PROCEEDINGS OF THE

AMERICAN MATHEMATICAL SOCIETY

Volume 132, Number 6, Pages 1675-1684

S 0002-9939(04)07385-X

Article electronically published on January 12, 2004

\title{
WEAK* PROPERTIES OF WEIGHTED CONVOLUTION ALGEBRAS
}

\author{
SANDY GRABINER
}

(Communicated by N. Tomczak-Jaegermann)

\begin{abstract}
Suppose that $L^{1}(\omega)$ is a weighted convolution algebra on $\mathbf{R}^{+}=$ $[0, \infty)$ with the weight $\omega(t)$ normalized so that the corresponding space $M(\omega)$ of measures is the dual space of the space $C_{0}(1 / \omega)$ of continuous functions. Suppose that $\phi: L^{1}(\omega) \rightarrow L^{1}\left(\omega^{\prime}\right)$ is a continuous nonzero homomorphism, where $L^{1}\left(\omega^{\prime}\right)$ is also a convolution algebra. If $L^{1}(\omega) * f$ is norm dense in $L^{1}(\omega)$, we show that $L^{1}\left(\omega^{\prime}\right) * \phi(f)$ is (relatively) weak ${ }^{*}$ dense in $L^{1}\left(\omega^{\prime}\right)$, and we identify the norm closure of $L^{1}\left(\omega^{\prime}\right) * \phi(f)$ with the convergence set for a particular semigroup. When $\phi$ is weak ${ }^{*}$ continuous it is enough for $L^{1}(\omega) * f$ to be weak* dense in $L^{1}(\omega)$. We also give sufficient conditions and characterizations of weak ${ }^{*}$ continuity of $\phi$. In addition, we show that, for all nonzero $f$ in $L^{1}(\omega)$, the sequence $f^{n} /\left\|f^{n}\right\|$ converges weak* to 0 . When $\omega$ is regulated, $f^{n+1} /\left\|f^{n}\right\|$ converges to 0 in norm.
\end{abstract}

\section{INTRODUCTION}

Suppose that $\omega(x)$ is a positive Borel measurable function on $\mathbf{R}^{+}=[0, \infty)$. When both $\omega$ and $1 / \omega$ are locally bounded on $[0, \infty)$, we say that $\omega$ is a weight. When $\omega(x)$ is a weight, then $L^{1}(\omega)$ is the Banach space of (equivalence classes of) locally integrable functions $f$ for which $f \omega$ is integrable. We give $L^{1}(\omega)$ the inherited norm

$$
\|f\|=\|f\|_{\omega}=\|f \omega\|_{1}=\int_{0}^{\infty}|f(t)| \omega(t) d t .
$$

Similarly, $M(\omega)$ is the analogous space of measures with the norm

$$
\|\mu\|=\|\mu\|_{\omega}=\int_{\mathbf{R}^{+}} \omega(t) d|\mu|(t),
$$

and $C_{0}(1 / \omega)$ is the space of continuous functions $h$ on $[0, \infty)$ for which

$$
\lim _{x \rightarrow \infty} h(x) / \omega(x)=0,
$$

with the norm

$$
\|h(x)\|=\|h / \omega\|_{\infty}=\sup \{h(x) / \omega(x)\}_{x \geq 0} .
$$

We are particularly interested in the case in which the weight $\omega$ is an algebra weight; that is, $\omega$ is submultiplicative (i.e., $\omega(x+y) \leq \omega(x) \omega(y)$ ), is everywhere

Received by the editors October 9, 2002.

2000 Mathematics Subject Classification. Primary 43A10, 43A20, 43A22, 46J45.

The research for this paper was done while the author enjoyed the gracious hospitality of the Australian National University in Canberra. 
right continuous, and has $\omega(0)=1$. The submultiplicativity implies that both $L^{1}(\omega)$ and $M(\omega)$ are Banach algebras under convolution, and that $L^{1}(\omega)$ is a closed ideal in $M(\omega)$ when we identify the function $f(t)$ with the measure $f(t) d t$. The other conditions guarantee that $M(\omega)$ is the dual space of the separable Banach space $C_{0}(1 / \omega)$ under the natural duality $\langle\mu, h\rangle=\int h(t) d \mu(t)$, Gr1, Theorem 2.2, p. 592] so that $M(\omega)$, and its subspace $L^{1}(\omega)$, are equipped with a natural weak* ${ }^{*}$ topology. Requiring that $\omega$ be an algebra weight in our sense is just a normalization. Whenever $L^{1}(\omega)$ is an algebra, we can always replace $\omega$ by an equivalent algebra weight without changing the space $L^{1}(\omega)$ or its norm topology [Gr1, Theorem 2.1, p. 591].

In this paper we examine the structure of $L^{1}(\omega)$, and particularly homomorphisms between such algebras, in the weak ${ }^{*}$ topology. The weak ${ }^{*}$ topology is in many ways better behaved than the norm topology, and, as we shall see, can be used as a tool in proving results for normed topologies.

In section 2, we collect results characterizing weak* convergence of bounded nets in $M(\omega)$ and relating weak* convergence to convergence in various norms. In sections 3 and 4 we consider continuous nonzero homomorphisms $\phi: L^{1}(\omega) \rightarrow$ $L^{1}\left(\omega^{\prime}\right)$. In previous papers, starting with GGM, we considered characterizations of sufficient conditions for $\phi$ to be what we called a standard homomorphism; that is, $L^{1}(\omega) * f$ being norm dense in $L^{1}(\omega)$ implies $L^{1}\left(\omega^{\prime}\right) * \phi(f)$ is norm dense in $L^{1}\left(\omega^{\prime}\right)$. In section 3 we show that $L^{1}\left(\omega^{\prime}\right) * \phi(f)$ is always weak* dense, and we describe the norm closure of $L^{1}\left(\omega^{\prime}\right) * \phi(f)$. When $\phi$ is weak ${ }^{*}$ continuous, we show that it is enough for $L^{1}(\omega) * f$ to be weak ${ }^{*}$ dense. In section 4 , we give characterizations and useful sufficient conditions for the weak* continuity of $\phi$. In section 5 we give conditions on $\omega$ that guarantee that the sequences $f^{(n+1)} /\left\|f^{n}\right\|$ converge to 0 in norm. This is done by first showing that $f^{n} /\left\|f^{n}\right\|$ always converges to zero weak*, and then applying results collected in section 2.

\section{WEAK* CONVERGENCE OF NETS}

We first collect, mostly from earlier papers, a number of equivalent characterizations of weak* convergence of nets in $M(\omega)$.

Theorem 2.1. Suppose that $\omega$ is an algebra weight on $\mathbf{R}^{+}$, and let $\left\{\lambda_{n}\right\}$ be a bounded net in $M(\omega)$. If $\left\{\lambda_{n}\right\}$ converges to $\lambda$ weak $k^{*}$ in $M(\omega)$, then we have:

(a) For all $\nu$ in $M(\omega)$, weak ${ }^{*}-\lim _{n} \lambda_{n} * \nu=\lambda * \nu$.

(b) For all continuous functions $f$ on $\mathbf{R}^{+}$with $f(0)=0$, the net $\left\{\lambda_{n} * f\right\}$ converges pointwise to $\lambda * f$.

(c) If $\omega^{\prime}$ is a weight with $\omega^{\prime} / \omega$ bounded and integrable and if $f$ belongs to $L^{1}(\omega)$, then $\left\{\lambda_{n} * f\right\}$ converges to $\lambda * f$ in the norm of $L^{1}\left(\omega^{\prime}\right)$.

(d) For all locally integrable $f$ and all $a>0, \lim _{n} \int_{0}^{a}\left|\lambda_{n} * f(t)-\lambda * f(t)\right| d t=0$.

Conversely, if one of the conditions (a), (b), (c), or (d) holds for a single nonzero function (or measure), then $\left\{\lambda_{n}\right\}$ converges weak $k^{*}$ to $\lambda$ in $M(\omega)$.

The proof that (a) is equivalent to weak* convergence is in Gr1 Lemma (2.2)]. Part (b) is proved in [GG1, Theorem (3.1)(a), p. 511]. The proof is given for sequences, but the same proof works for nets. Part (c), for sequences, is GG1, Theorem (3.2), p. 512]; but [GG3. Theorem (1.3)] shows that if (c) holds for some $f$ and all weak* convergent sequences, then convolution by $f$ is a compact operator 
from $M(\omega)$ to $L^{1}\left(\omega^{\prime}\right)$. This then implies that (c) also holds for all bounded weak* convergent nets. Part (d) is an easy consequence of (c) [GG1, Cor. (3.3), p. 513].

Once we know that some type of convergence, as in (a), (b), (c), or (d), follows from weak ${ }^{*}$ convergence, the proof of the converse follows from the fact that every bounded net has a weak* convergent subnet, together with the fact that the convolution of nonzero measures on $\mathbf{R}^{+}$can never be zero. For the details see [Gr1] Lemma 3.2, p. 595], or [Gr3, Theorem (4.1), p. 183].

There are numerous other useful characterizations of weak* convergence. For instance [GG2, p. 52], it is enough that $\lim _{n}\left\langle\lambda_{n}, h\right\rangle=\langle\lambda, h\rangle$ for all continuous $h$ with compact support, since the set functions with compact support are dense in $C_{0}(1 / \omega)$.

The nicest results occur when all $\lambda_{n} * f$ converge to $\lambda * f$ in the norm of $L^{1}(\omega)$. Recall that the algebra weight $\omega(t)$ is regulated at $b \geq 0$ if $\lim _{x \rightarrow \infty} \omega(x+a) / \omega(x)=0$ for all $a>b$. Recall also that if $\lambda$ is a locally integrable function or a locally finite measure of $\mathbf{R}^{+}$, then $\alpha(\lambda)$ is the infimum of the support of $\lambda(\alpha(0)=\infty)$. The basic result relating convergence to weak* convergence in $M(\omega)$ is the following result, taken from [GGM, Theorem (3.2), p. 284] and [GG1, Theorem (2.3), p. 509].

Theorem 2.2. Suppose that $\omega$ is an algebra weight on $\mathbf{R}^{+}$and that $b \geq 0$. Then the following are equivalent:

(a) $\omega$ is regulated at $b$.

(b) Whenever $\left\{\lambda_{n}\right\}$ is a bounded net converging weak $k^{*}$ to $\lambda$ in $M(\omega)$ and $g$ is a function in $L^{1}(\omega)$ with $\alpha(g) \geq b$, then $\lambda_{n} * g$ converges to $\lambda * g$ in the norm of $L^{1}(\omega)$.

Condition (c) in Theorem (2.1) is the simplest condition on $\omega^{\prime} / \omega$ that guarantees convergence in norm in $L^{1}\left(\omega^{\prime}\right)$. A determination of precisely which $\omega^{\prime} / \omega$ work is given in GG3. The most important case, $\omega^{\prime}=\omega$, is Theorem (2.2) above.

Theorem (2.1)(a) says that multiplication is weak* separately continuous on bounded subsets of $M(\omega)$. In fact, it is not hard to show [Gr1 Lemma 3.1, p. 595] that multiplication is weak ${ }^{*}$ separately continuous on all of $M(\omega)$. The following result shows that on bounded subsets of $M(\omega)$, multiplication is actually jointly continuous in the weak* topology. Since the weak topology restricted to bounded subsets of $M(\omega)=C_{0}(1 / \omega)^{*}$ is metrizable [DS, Theorem V.5.1, p. 426], we need only consider sequences in the following result.

Theorem 2.3. Suppose that $\omega$ is an algebra weight and that $\left\{\lambda_{n}\right\}$ and $\left\{\mu_{n}\right\}$ are sequences in $M(\omega)$. If weak $k^{*}-\lim \lambda_{n}=\lambda$ and weak ${ }^{*}-\lim \mu_{n}=\mu$, then weak ${ }^{*}$ $\lim \lambda_{n} * \mu_{n}=\lambda * \mu$.

Proof. Choose some nonzero $f$ in $L^{1}(\omega)$, and let $\omega^{\prime}$ be as in Theorem (2.1)(c); for instance, we could let $\omega^{\prime}(t)=e^{-t} \omega(t)$. Then $\lambda_{n} * f \rightarrow \lambda * f$ and $\mu_{n} * f \rightarrow \mu * f$ in norm in the Banach algebra $L^{1}\left(\omega^{\prime}\right)$. Hence the $L^{1}\left(\omega^{\prime}\right)$ norm limit of $\left(\lambda_{n} * \mu_{n}\right) *(f * f)$ is $(\lambda * \mu) *(f * f)$. It then follows from Theorem (2.1) that $\lambda_{n} * \mu_{n} \rightarrow \lambda * \mu$ in the weak* topology on $M(\omega)$.

\section{WEAK*-STANDARD HOMOMORPHISMS}

Throughout this section, $\omega$ and $\omega^{\prime}$ are algebra weights and $\phi: L^{1}(\omega) \rightarrow L^{1}\left(\omega^{\prime}\right)$ is a continuous nonzero homomorphism. Then $\phi$ has a unique extension to a homomorphism from $M(\omega)$ to $M\left(\omega^{\prime}\right)$, and this extension is continuous with the same 
norm [Gr1, Theorem 3.4, p. 596]. Because of the uniqueness, we let $\phi$ denote both the original map and its extension. The homomorphism $\phi$ is said to be standard GGM, p. 278] if $L^{1}\left(\omega^{\prime}\right) * \phi(f)$ is norm dense in $L^{1}\left(\omega^{\prime}\right)$ whenever $L^{1}(\omega) * f$ is norm dense in $L^{1}(\omega)$. In [GGM] we gave several equivalent characterizations of the standardness of homomorphisms, and we showed [GGM], Theorem (3.4), p. 284] that $\phi$ is standard if $\omega^{\prime}$ is regulated at any $b \geq 0$; that is, if $\lim _{x \rightarrow \infty} \omega^{\prime}(x+a) / \omega^{\prime}(x)=0$ for any $a>0$. In this section we show that $L^{1}\left(\omega^{\prime}\right) * \phi(f)$ is always weak* dense when $L^{1}(\omega) * f$ is norm dense.

Let $\left\{\delta_{t}\right\}_{t \geq 0}$ be the convolution semigroup of point masses, so that $\delta_{t} * f(x)=$ $f(x-t)$, the right translation of $f$; and let $\mu_{t}=\phi\left(\delta_{t}\right)$. Following GGM, p. 280], we call

$$
I=\left\{g \in L^{1}\left(\omega^{\prime}\right): \lim _{t \rightarrow 0} \mu_{t} * g=g\right\}
$$

the convergence ideal of $\phi$ (or of the semigroup $\left\{\mu_{t}\right\}$ ). Since $\left\{\mu_{t}\right\}$ is norm bounded near 0 , the set $I$ is easily seen to be a closed ideal. One of the characterizations of standardness of $\phi$ is that $I=L^{1}\left(\omega^{\prime}\right)$; that is, that (convolution by) $\mu_{t}$ is a strongly continuous semigroup on $L^{1}\left(\omega^{\prime}\right)$ GGM, Theorem (2.2)(a), p. 280]. We are now ready for our main result.

Theorem 3.1. Suppose that $\omega$ and $\omega^{\prime}$ are algebra weights and that $\phi: L^{1}(\omega) \rightarrow$ $L^{1}\left(\omega^{\prime}\right)$ is a continuous nonzero homomorphism. If $L^{1}(\omega) * f$ is norm dense in $L^{1}(\omega)$, then we have:

(a) The norm closure of $L^{1}\left(\omega^{\prime}\right) * \phi(f)$ is the convergence ideal of $\phi$.

(b) $L^{1}\left(\omega^{\prime}\right) * \phi(f)$ is weak $k^{*}$ dense in $L^{1}\left(\omega^{\prime}\right)$.

Proof. Since we know [Gr2] Corollary (2.5), p. 162] that the convergence ideal of $\phi$ is weak* dense, it will be enough to prove (a). We also know GGM, Theorem (2.4), pp. 281-282] that there exists $f$, specifically $f(t)=e^{-r t}$, with $L^{1}(\omega) * f$ dense and the norm closure $L^{1}\left(\omega^{\prime}\right) * \phi(f)$ equalling the convergence ideal of $\phi$. It is also easy to see that all $L^{1}\left(\omega^{\prime}\right) * \phi(g)$ belong to the convergence ideal of $\phi$ GGM, p. 282]. To complete the proof to the theorem, we just need the following lemma.

Lemma 3.2. If $L^{1}(\omega) * f$ is norm dense in $L^{1}(\omega)$, then the norm closure of $L^{1}\left(\omega^{\prime}\right) *$ $\phi(f)$ contains the norm closure of $L^{1}\left(\omega^{\prime}\right) * \phi(g)$ for all $g$ in $L^{1}(\omega)$.

Proof. Since $L^{1}(\omega) * f$ is dense, we can find a sequence $\left\{h_{n}\right\}$ in $L^{1}(\omega)$ for which $\lim \left(f * h_{n}\right)=g$, with the limit taken in the norm topology. By the continuity of $\phi$, this implies that $\lim \phi(f) * \phi\left(h_{n}\right)=\phi(g)$. Hence $\phi(g)$, and therefore the norm closure of $L^{1}\left(\omega^{\prime}\right) * \phi(g)$ as well, belongs to the norm closure of $L^{1}\left(\omega^{\prime}\right) * \phi(f)$. This completes the proof of the lemma, and of Theorem (3.1).

Notice that not only the theorem, but also the lemma, show that the norm closure of $L^{1}\left(\omega^{\prime}\right) * \phi(f)$ is the same for all $f$ in $L^{1}(\omega)$ with $L^{1}(\omega) * f$ norm dense. This greatly simplifies the formulas we were able to obtain in [GGM, p. 282].

The natural weak* analogue of standardness of homomorphisms should only assume that $L^{1}(\omega) * f$ is weak* dense in $L^{1}(\omega)$, rather than norm dense. The next result shows that this natural analogue does hold when the homomorphism is weak* continuous rather than just norm continuous. In section 4 , we will study when homomorphisms are weak* continuous. 
Theorem 3.3. Suppose that the nonzero homomorphism $\phi: L^{1}(\omega) \rightarrow L^{1}\left(\omega^{\prime}\right)$ is weak $k^{*}$ continuous. If $L^{1}(\omega) * f$ is weak $k^{*}$ dense in $L^{1}(\omega)$, then $L^{1}\left(\omega^{\prime}\right) * \phi(f)$ is weak $k^{*}$ dense in $L^{1}\left(\omega^{\prime}\right)$.

Proof. Choose some $g$, say $g=e^{-r t}$, with $L^{1}(\omega) * g$ norm dense. Since we already know that $L^{1}\left(\omega^{\prime}\right) * \phi(g)$ is weak* dense, it will be enough to show that $\phi(g)$ is the weak ${ }^{*}$ limit of a sequence in $L^{1}\left(\omega^{\prime}\right) * \phi(f)$. Since $L^{1}(\omega) * f$ is weak ${ }^{*}$ dense, we can find a sequence $\left\{h_{n}\right\}$ in $L^{1}(\omega)$ with weak* $\lim \left(f * h_{n}\right)=g$. Since $\phi$ is a weak* continuous homomorphism, this shows that $\phi\left(f * h_{n}\right)=\phi(f) * \phi\left(h_{n}\right)$ converges weak ${ }^{*}$ to $\phi(g)$ in $L^{1}\left(\omega^{\prime}\right)$. This completes the proof.

The major unsolved question in the ideal theory of radical $L^{1}(\omega)$ is the standard ideal problem, which asks if $L^{1}(\omega) * f$ must be norm dense for all $f$ in $L^{1}(\omega)$ with $\alpha(f)=0$ ([D, p. 557], and [GG1, Question 1, p. 507]). The results in this section suggest the following, presumably easier, weak* analogue.

Question 3.4. Suppose that $L^{1}(\omega)$ is a radical algebra and that $f$ in $L^{1}(\omega)$ has $\alpha(f)=0$. Must $L^{1}(\omega) * f$ be weak $k^{*}$ dense in $L^{1}(\omega)$ ?

When $\omega$ is regulated at any $b \geq 0, L^{1}(\omega) * f$ is norm dense if it is weak* dense (GG3, Theorem (5.1)(b)] and [BD, Proposition 1.9, p. 72]). Hence an affirmative answer to Question (3.4) would solve the standard ideal problem for regulated weights. Of course a negative answer to Question (3.4) would also be a negative answer to the standard ideal problem.

\section{WEAK* CONTINUOUS HOMOMORPHISMS}

As in the previous section, we let $\phi: L^{1}(\omega) \rightarrow L^{1}\left(\omega^{\prime}\right)$ be a continuous nonzero homomorphism, where $\omega$ and $\omega^{\prime}$ are algebra weights. In this section we give sufficient conditions and characterizations of $\phi$ being weak* continuous. We start with some preliminary results. The following result is essentially a variant of the Krein-Smulian Theorem.

Lemma 4.1. Let $E$ and $F$ be Banach spaces, and let $T: F^{*} \rightarrow E^{*}$ be a linear map. Then $T$ is weak $k^{*}$ continuous if weak $k^{*} \lim T\left(\lambda_{n}\right)=T(\lambda)$ whenever $\left\{\lambda_{n}\right\}$ is a bounded net with weak* limit $\lambda$. When $F$ is separable, it is enough to consider only bounded sequences.

Proof. It follows from the Krein-Smulian Theorem [DS, Theorem V.5.7, p. 429] that it is enough to show that the restriction of $T$ to closed balls is weak* continuous. But this translates to the statement about bounded nets in the theorem. When $F$ is separable, then the weak ${ }^{*}$ topology on closed balls of $F^{*}$ is metrizable DS. Theorem V.5.1, p. 426]. So one only needs to consider sequences to prove continuity.

As one application of the above lemma we show that if $\phi$ is weak* continuous, then so is its extension to the corresponding measure algebras, just as with norm continuity.

Lemma 4.2. If $\phi: L^{1}(\omega) \rightarrow L^{1}\left(\omega^{\prime}\right)$ is a nonzero weak* continuous homomorphism, then so is its extension to a homomorphism from $M(\omega)$ to $M\left(\omega^{\prime}\right)$.

Proof. Let $\left\{\lambda_{n}\right\}$ be a bounded sequence (or net) in $M(\omega)$ with weak* limit $\lambda$. Let $f$ be a nonzero element of $L^{1}(\omega)$ with $\phi(f) \neq 0$. Then, by Theorem $(2.1), \lambda_{n} * f$ converges weak ${ }^{*}$ to $\lambda * f$ in $L^{1}(\omega)$. By the weak* continuity of $\phi$ on $L^{1}(\omega)$, this 
means that $\phi\left(\lambda_{n} * f\right)=\phi\left(\lambda_{n}\right) * \phi(f)$ converges weak* to $\phi(\lambda) * \phi(f)$. Since a weak* continuous map, like $\phi$, must also be norm continuous, the sequence $\left\{\phi\left(\lambda_{n}\right)\right\}$ is bounded. It then follows, from Theorem (2.1) again, that weak*-lim $\phi\left(\lambda_{n}\right)=\phi(\lambda)$. By Lemma (4.1), this implies that $\phi: M(\omega) \rightarrow M\left(\omega^{\prime}\right)$ is weak* continuous, and thus completes the proof.

We will need the following simple result in both this and the next section.

Lemma 4.3. Suppose that $\left\{\lambda_{n}\right\}$ is a net in $M(\omega)$. If $\lim \alpha\left(\lambda_{n}\right)=\infty$, then we have

(a) $\left|\lambda_{n}\right|([0, a)) \rightarrow 0$ for all $a>0$.

(b) If $\left\{\lambda_{n}\right\}$ is bounded, then weak $k^{*} \lim \lambda_{n}=0$.

Proof. Part (a) is clear, since $\left|\lambda_{n}\right|([0, a))=0$ for all sufficiently large $n$. Similarly, if $h$ is a continuous function with compact support, $\lim \left\langle\lambda_{n}, h\right\rangle=0$. When $\left\{\lambda_{n}\right\}$ is bounded, the weak ${ }^{*}$-convergence of $\left\{\lambda_{n}\right\}$ then follows from the remarks after the proof of Theorem (2.1).

The convergence in part (a) is much stronger than weak* convergence. For instance, $\delta_{1 / n}-\delta_{0}$ converges to 0 weak $^{*}$ in every $M(\omega)$, but $\left|\delta_{1 / n}-\delta_{0}\right|([0, a))=2$ for every $a>1$.

We now give two different sufficient conditions for weak ${ }^{*}$ continuity of $\phi$ : $L^{1}(\omega) \rightarrow L^{1}\left(\omega^{\prime}\right)$. These results are in part motivated by Theorem (3.3) above, which essentially says that weak* continuous homomorphisms are weak* ${ }^{*}$ standard. First we give a simple proof of our earlier result [GGM, Theorem (3.5), p. 285], which says that $\phi$ is weak* continuous if $\omega$ is regulated at any $b \geq 0$. In this case Theorem (3.3) does not improve on Theorem (3.1) because, when $\omega$ is regulated, if $L^{1}(\omega) * f$ is weak* dense, then it must also be norm dense [GG3, Theorem (5.1)(b)].

Theorem 4.4. Suppose that $\omega$ and $\omega^{\prime}$ are algebra weights. If $\omega$ is regulated at some $b \geq 0$, then every continuous homomorphism $\phi: L^{1}(\omega) \rightarrow L^{1}\left(\omega^{\prime}\right)$ is weak $k^{*}$ continuous.

Proof. Without loss of generality, we can assume that $\phi$ is not the zero homomorphism, so that we can apply Lemma (4.1). Let $\left\{\lambda_{n}\right\}$ be a bounded sequence (or net) in $M(\omega)$ with weak ${ }^{*} \lim \lambda_{n}=\lambda$. Choose $g$ in $L^{1}(\omega)$ with $\phi(g) \neq 0$ and $\alpha(g) \geq b$ (for instance, choose any $f$ with $\phi(f) \neq 0$ and let $g=\delta_{b} * f$ ). By Theorem (2.2), $\lambda_{n} * g \rightarrow \lambda * g$ in the norm of $L^{1}(\omega)$. Since $\phi$ is norm continuous, this implies that $\phi\left(\lambda_{n} * g\right)=\phi\left(\lambda_{n}\right) * \phi(g)$ converges to $\lambda * g$ in norm and hence weak*. It then follows from Theorem (2.1) that $\phi\left(\lambda_{n}\right) \rightarrow \phi(\lambda)$ weak* ${ }^{*}$ The theorem now follows from Lemma (4.1).

For our other sufficient condition for weak* continuity, we will need to recall some terminology and results from [Gr1. Suppose that $\phi: L^{1}(\omega) \rightarrow L^{1}\left(\omega^{\prime}\right)$ is a nonzero homomorphism, and let $\mu_{t}=\phi\left(\delta_{t}\right)$. Then there is a nonnegative number $A$ for which $\alpha\left(\mu_{t}\right)=A t$ [Gr1, Theorem (4.3)(a), p. 605], GhM, Lemma 1, p. 344]. We call $A$ the character of $\phi$, and of $\left\{\mu_{t}\right\}$. When the character $A$ is strictly positive, one also has [Gr1, Theorem 4.9, p. 607] $\alpha(\phi(\lambda))=A \alpha(\lambda)$ for all $\lambda$ in $M(\omega)$.

In our proof of the weak* continuity of homomorphisms of positive character, we will use Theorem $(2.1)(\mathrm{d})$. The following notation will be convenient for this purpose. For each $a>0$, we define the seminorm $\|f\|_{a}=\int_{0}^{a}|f(t)| d t$ on $L_{l o c}^{1}$, the space of locally integrable functions on $\mathbf{R}^{+}$. Thus Theorem (2.1)(d) says that $\lambda_{n} * f$ converges to $\lambda * f$ in each of these seminorms. Similarly, Lemma (4.3)(a) says that 
$\lambda_{n} \rightarrow 0$ in the analogous seminorms on $M_{l o c}\left(\mathbf{R}^{+}\right)$, the space of locally finite Borel measures on $\mathbf{R}^{+}=[0, \infty)$. We can now prove:

Theorem 4.5. Let $\phi: L^{1}(\omega) \rightarrow L^{1}\left(\omega^{\prime}\right)$ be a continuous nonzero homomorphism. If the character $A$ of $\phi$ is strictly positive, then $\phi$ is weak* continuous.

Proof. We first show that for each $a>0$ there is an $M=M(a)>0$ for which

$$
\|\phi(f)\|_{A a} \leq M\|f\|_{a}
$$

for each $f$ in $L^{1}(\omega)$. Let $L^{1}(\omega)_{a}=\left\{f \in L^{1}(\omega): \alpha(f) \geq a\right\}=\left\{f \in L^{1}(\omega):\|f\|_{a}=\right.$ $0\}$, and define $L^{1}\left(\omega^{\prime}\right)_{A a}$ analogously. Since $\phi\left(L^{1}(\omega)_{a}\right) \subseteq L^{1}\left(\omega^{\prime}\right)_{A a}$, it follows that $\phi$ induces a continuous map from the quotient Banach algebra $L^{1}(\omega) / L^{1}(\omega)_{a}$ to the quotient algebra $L^{1}\left(\omega^{\prime}\right) / L^{1}\left(\omega^{\prime}\right)_{A a}$. Since $\omega$ is bounded and bounded below on $[0, a)$, the quotient norm is equivalent to the norm induced by the seminorm $f \mapsto\|f\|_{a}$, and similarly for the norm induced by the seminorm $g \mapsto\|g\|_{A a}$ on the quotient of $L^{1}\left(\omega^{\prime}\right)$. Formula (4.1) is now just the statement that the map induced by $\phi$ between the quotient spaces is bounded.

Now suppose that $\lambda_{n}$ is a bounded sequence or net in $M(\omega)$ that converges weak* to $\lambda$. Choose $f \in L^{1}(\omega)$ with $\phi(f) \neq 0$ (actually $\phi$ has kernel $\{0\}$ Gr1. Appendix, p. 613]). By Theorem (2.1)(d), each $\left\|\lambda_{n} * f-\lambda * f\right\|_{a}$ converges to 0 . By formula (4.1), this implies that each $\left\|\phi\left(\lambda_{n}\right) * \phi(f)-\phi(\lambda) * \phi(f)\right\|_{b}$ converges to

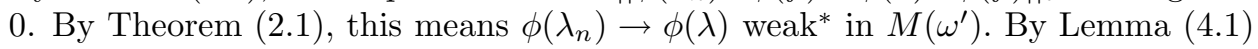
this implies that $\phi$ is weak* continuous, and therefore completes the proof of the theorem.

The algebra $L_{l o c}^{1}\left(\mathbf{R}^{+}\right)$of locally integrable functions on $\mathbf{R}^{+}$is a Fréchet algebra under the seminorms $\|\cdot\|_{a}$ for $a>0$. Thus Theorem (2.1)(d) says that $\lambda_{n} * f$ converges to $\lambda * f$ in the Fréchet topology on $L_{l o c}^{1}\left(\mathbf{R}^{+}\right)$, and formula (4.1) says that $\phi$ is continuous in this topology, relativized by $L^{1}(\omega)$ and $L^{1}\left(\omega^{\prime}\right)$. The algebra $L_{l o c}^{1}\left(\mathbf{R}^{+}\right)$, and particularly its automorphisms and derivations, is studied by Ghahramani and McClure in GhM]. In a paper in preparation I will study the continuous homomorphisms of $L_{l o c}^{1}\left(\mathbf{R}^{+}\right)$.

Homomorphisms of positive character seem to be better behaved than homomorphisms of character 0. Thus for positive character $A$ we have $\alpha(\phi(\mu))=A \alpha(\mu)$, which implies that $\phi$ is one-to-one [Gr1, Appendix, p. 613]. By the previous theorem and its proof we also know that $\phi$ is weak* continuous (so that Theorem (3.3) applies) and is continuous in the (relativized) Fréchet topology on $L_{l o c}^{1}\left(\mathbf{R}^{+}\right)$. While much is known for character 0 [Gr1], the following natural question is open (for partial results see [Gr1, Theorem 4.11, p. 608]).

Question 4.6. If $\phi: L^{1}(\omega) \rightarrow L^{1}\left(\omega^{\prime}\right)$ has character 0 , must $\alpha(\phi(\mu))=0$ for all $\mu$ in $M(\omega)$ ?

If the answer to Question (4.6) is yes, then $\phi$ would be one-to-one, and hence all continuous nonzero homomorphisms $\phi: L^{1}(\omega) \rightarrow L^{1}\left(\omega^{\prime}\right)$ would be one-to-one. For a discussion of known results on when $\phi$ is one-to-one, see [Gr3, Section 5].

We now give a relatively simple characterization of weak* continuity of homomorphisms.

Theorem 4.7. Suppose that $\omega$ and $\omega^{\prime}$ are algebra weights and that $\phi: L^{1}(\omega) \rightarrow$ $L^{1}\left(\omega^{\prime}\right)$ is continuous, and let $\mu_{t}=\phi\left(\delta_{t}\right)$. Then $\phi$ is weak* continuous if and only if




We first separate out the direction that assumes that $\phi$ is weak* continuous, and we determine the pre-adjoint of $\phi$ in this case.

Theorem 4.8. Suppose that $\phi: L^{1}(\omega) \rightarrow L^{1}\left(\omega^{\prime}\right)$ is a weak $k^{*}$-continuous nonzero homomorphism. Then we have

(a) $w e a k^{*}-\lim _{x \rightarrow \infty} \mu_{x} / \omega(x)=0$;

(b) $\phi$ is the adjoint of the map $T: C_{0}\left(1 / \omega^{\prime}\right) \rightarrow C_{0}(1 / \omega)$ given by $T h(x)=$ $\left\langle\mu_{x}, h\right\rangle$.

Proof. We first observe that we always have weak*- $\lim _{x \rightarrow \infty} \delta_{x} / \omega(x)=0$ in $M(\omega)$. For suppose that $h$ belongs to the predual $C_{0}(1 / \omega)$. Then

$$
\left\langle\delta_{x} / \omega(x), h\right\rangle=h(x) / \omega(x) \rightarrow 0 \quad \text { as } x \rightarrow \infty,
$$

by the definition of $C_{0}(1 / \omega)$. Now when $\phi$ is weak ${ }^{*}$ continuous we therefore have that $\phi\left(\delta_{x} / \omega(x)\right)=\mu_{x} / \omega(x)$ has weak* limit 0 as $x$ goes to $\infty$. This proves (a).

Since $\phi$ is weak ${ }^{*}$ continuous, there is some bounded linear map $L: C_{0}\left(1 / \omega^{\prime}\right) \rightarrow$ $C_{0}(1 / \omega)$ with $\phi=L^{*}$. We just need to show that $L$ equals $T$ as defined in (b). Choose an $h$ in $C_{0}\left(1 / \omega^{\prime}\right)$. Then for all $x \geq 0$ we have

$$
L h(x)=\left\langle\delta_{x}, L h\right\rangle=\left\langle L^{*} \delta_{x}, h\right\rangle=\left\langle\phi\left(\delta_{x}\right), h\right\rangle=T h(x),
$$

as required. This completes the proof of Theorem (4.8).

Proof of Theorem (4.7). Suppose that $\mu_{x} / \omega(x)$ approaches 0 weak $^{*}$ as $x \rightarrow \infty$. We need to show that $\phi$ is weak* continuous. We do this by first showing that the map $T$ of Theorem (4.8)(b) is a bounded linear map, and then showing that $\phi=T^{*}$.

For $h$ in $C_{0}\left(1 / \omega^{\prime}\right)$ we define the function $T h$ on $\mathbf{R}^{+}$by $T h(x)=\left\langle\mu_{x}, h\right\rangle$. Since $\mu_{x}$ is weak* continuous [Gr1, Theorem 3.6(A), p. 599], Th is a continuous function. Since $T h(x) / \omega(x)=\left\langle\mu_{x} / \omega(x), h\right\rangle$, we have $T h$ in $C_{0}(1 / \omega)$ by our assumption that $\mu_{x} / \omega(x) \rightarrow 0$ weak*. We now need to show that the linear map $T: C_{0}\left(1 / \omega^{\prime}\right) \rightarrow$ $C_{0}(1 / \omega)$ is bounded. Since all $\delta_{x} / \omega(x)$ are unit vectors in $L^{1}(\omega)$, we have, for each $x \geq 0$, that

$$
|T h(x) / \omega(x)| \leq \frac{\left\|\mu_{x}\right\|}{\omega(x)}\|h\| \leq\|\phi\|\|h\|,
$$

where the norm of $h$ is taken in $C_{0}\left(1 / \omega^{\prime}\right)$. Thus

$$
\|T h\|=\sup |T h(x) / \omega(x)| \leq\|\phi\|\|h\|,
$$

so that $T$ is bounded.

Now, for each $f$ in $L^{1}(\omega)$, we have $\phi(f)=\int_{0}^{\infty} f(t) \mu_{t} d t$ as a weak* integral on $L^{1}\left(\omega^{\prime}\right)$ Gr1 formula (3.7), p. 599]. This means that for each $h$ in $C_{0}\left(1 / \omega^{\prime}\right)$, we have

$$
\langle\phi f, h\rangle=\int_{0}^{\infty} f(t)\left\langle\mu_{t}, h\right\rangle d t=\int_{0}^{\infty} f(t) T h(t)=\langle f, T h\rangle=\left\langle T^{*} f, h\right\rangle .
$$

Thus $\phi=T^{*}$; so $\phi$ is weak* continuous. This completes the proof of Theorem (4.7).

Verifying the condition that $\mu_{x} / \omega(x) \rightarrow 0$ weak* should usually be easier to do than directly proving that $\phi$ is weak ${ }^{*}$ continuous. For instance, if $\phi: L^{1}(\omega) \rightarrow$ $L^{1}\left(\omega^{\prime}\right)$ is a continuous homomorphism with positive character, then $\mu_{x} / \omega(x)$ is a bounded net with $\lim _{x \rightarrow \infty} \alpha\left(\mu_{x} / \omega(x)\right)=0$. It then follows from Lemma (4.3) that $\mu_{x} / \omega(x) \rightarrow 0$ weak $^{*}$ as $x$ goes to $\infty$. 


\section{NORMALIZED POWERS}

There have been several papers which have considered the sequences $\omega_{n}=\left\|f^{n}\right\|$ of norms of powers and the sequence $f^{n} /\left\|f^{n}\right\|$ of normalized powers of elements $f$ of radical Banach algebras. See [A], [W], [S], [LRRW]. There are two extreme cases LRRW, Corollary 2.5]. Solovej [S] showed that for $f$ in the Volterra algebra $L^{1}[0,1)$ with $\alpha(f)=0$, we always have $\lim \omega_{n+1} / \omega_{n}=0$, so that the sequence $\left\{\omega_{n}\right\}$ is regulated at 1 in the sense of [BDL. Loy, et al. LRRW construct and study $f$ for which $f^{n} /\left\|f^{n}\right\|$ has a subsequence that is a bounded approximate identity. This is the key part of their construction of a weakly amenable commutative radical Banach algebra. We show that the situation in $L^{1}(\omega)$ is closer to the first extreme. We always have weak*- $\lim \left(f^{n} /\left\|f^{n}\right\|\right)=0$, and when $\omega(t)$ is regulated we also have $\lim \left(f^{n+1} /\left\|f^{n}\right\|\right)=0$.

Theorem 5.1. Suppose that $\omega$ is an algebra weight. For all nonzero $f$ in $L^{1}(\omega)$, the sequence $f^{n} /\left\|f^{n}\right\|$ converges to 0 in the weak $k^{*}$ topology of $L^{1}(\omega)$.

Proof. To simplify the notation, we let $g_{n}=f^{n} /\left\|f^{n}\right\|$. If $\alpha(f)>0$, then $\lim \alpha\left(g_{n}\right)=$ 0 ; so $g_{n} \rightarrow 0$ weak $^{*}$ by Lemma $(4.3)(\mathrm{b})$. Now suppose that $\alpha(f)=0$. If $\omega(x) \geq C$ on $[0, a)$, then $\left\|f^{n}\right\|_{\omega} \geq C\left\|f^{n}\right\|_{a}$. Hence it follows easily from Solovej's result [S], and its obvious generalization to all $L^{1}[0, a)$ that $\left\|g_{n} * f\right\|_{a}=\int_{0}^{a}\left|g_{n} * f(t)\right| d t$ converges to 0 for all $a>0$. Then $g_{n} \rightarrow 0$ weak* $^{*}$, by Theorem $(2.1)$.

A direct application of Theorem (2.2) yields the following corollary.

Corollary 5.2. Suppose that $\omega(t)$ is an algebra weight that is regulated at $b \geq 0$. For all $f$ in $L^{1}(\omega)$ with $\alpha(f) \geq b$, we have $\lim \left(\frac{f^{n+1}}{\left\|f^{n}\right\|}\right)=0$.

\section{REFERENCES}

[A] G. R. Allan, An inequality involving product measures, in J. M. Bachar et al. (eds.), Radical Banach algebras and automatic continuity, 277-279, Lecture Notes in Math. \#975, Springer-Verlag, New York, 1983. MR 84m:46062

[BD] W. G. Bade and H. G. Dales, Continuity of derivations from radical convolution algebras, Studia Math. 95 (1989), 59-91. MR 90k:46115

[BDL] W. G. Bade, H. G. Dales, and K. B. Laursen, Multipliers of radical Banach algebras of power series, Mem. Amer. Math. Soc., 49, 1984. MR 85j:46094

[D] H. G. Dales, Banach algebras and automatic continuity, London Math. Soc. Monographs, 24, Clarendon Press, Oxford, 2000. MR 2002e:46001

[DS] N. Dunford and J. T. Schwartz, Linear operators, Part I, Wiley Interscience, New York, 1958. MR 22:8302

[Gh] F. Ghahramani, Isomorphisms between radical weighted convolution algebras, Proc. Edinburgh Math. Soc. (2) 26 (1983), 343-351. MR 85h:43002

[GG1] F. Ghahramani and S. Grabiner, Standard homomorphisms and convergent sequences in weighted convolution algebras, Illinois J. Math. 36 (1992), 505-527. MR 93d:46089

[GG2] F. Ghahramani and S. Grabiner, The $L^{P}$ theory of standard homomorphisms, Pacific J. Math. 168 (1995), 49-60. MR 96e:43004

[GG3] F. Ghahramani and S. Grabiner, Convergence factors and compactness in weighted convolution algebras, Canad. J. Math. 54 (2002), 303-323.

[GGM] F. Ghahramani, S. Grabiner, and J. P. McClure, Standard homomorphisms and regulated weights on weighted convolution algebras, J. Functional Anal. 91 (1990), 278-286. MR 91k:43007

[GhM] F. Ghahramani and J. P. McClure, Automorphisms and derivations of a Fréchet algebra of locally integrable functions, Studia Math. 103 (1992), 51-69. MR 93j:46055

[Gr1] S. Grabiner, Homomorphisms and semigroups in weighted convolution algebras, Indiana Univ. Math. J. 37 (1988), 589-615. MR 90f:43007 
[Gr2] S. Grabiner, Semigroups and the structure of weighted convolution algebras, in Proceedings of the Conference on Automatic Continuity and Banach Algebras, R. J. Loy, ed., Proc. Centre Math. Anal., Australian National University, vol. 21 (1989), 155-169. MR 91c: 43004

[Gr3] S. Grabiner, Weighted convolution algebras and their homomorphisms, in Functional Analysis and Operator Theory, Banach Center Publications 30 (1994), 175-190, Polish Acad. of Sci., Warsaw. MR 95e:43004

[HP] E. Hille and R. S. Phillips, Functional analysis and semi-groups, Amer. Math. Soc. Colloquium Publ. 31, Providence, R.I., 1957. MR 19:664d

[LRRW] R. J. Loy, C. J. Read, V. Runde, and G. A. Willis, Amenable and weakly amenable Banach algebras with compact multiplication, J. Functional Analysis 171 (2000), 78114. MR 2001h: 46088

[S] M. Solovej, Norms of powers in the Volterra algebra, Bull. Austral. Math. Soc. 50 (1994), 55-57. MR 95g:46101

[W] G. A. Willis, The norms of powers of functions in the Volterra algebra, in J. M. Bachar et al. (eds.), Radical Banach algebras and automatic continuity, 345-349, Lecture Notes in Math. \#975, Springer-Verlag, New York, 1983. MR 84m:46063

Department of Mathematics, Pomona College, Claremont, California 91711 